\title{
The Evolution of the Single-Nanocrystal
}

\section{Fluorescence Linewidth with Size and Shell: \\ Implications for Exciton-Phonon Coupling and the Optimization of Spectral Linewidths \\ Supporting Information}

Jian Cui, Andrew P. Beyler, Igor Coropceanu, Liam Cleary, Thomas R. Avila, Yue Chen, José M. Cordero, S. Leigh Heathcote, Daniel K. Harris, Ou Chen, Jianshu Cao, and Moungi G. Bawendi*

Department of Chemistry, Massachusetts Institute of Technology

Cambridge, Massachusetts 02139, USA

E-mail: mgb@mit.edu

*To whom correspondence should be addressed 


\section{Nanocrystal Synthesis}

\section{Chemicals}

1-octadecene (ODE, 90\%), trioctylphosphine oxide (TOPO 99\%), trioctylphosphine (TOP, $97 \%$ ), decylamine $(99 \%)$, and hexamethyldisilathiane $\left[(\mathrm{TMS})_{2} \mathrm{~S}\right.$, synthesis grade] were obtained from Sigma-Aldrich. Cadmium oxide (CdO, 99.998\%), selenium powder (99.999\%), octadecylphosphonic acid (ODPA, 97\%), and n-hexylphosphonic acid were purchased from Alfa Aesar. Diethylzinc (ZnEt $2, \geq 52$ wt. \% Zn basis) and dimethylcadmium ( $\left.\mathrm{CdMe}_{2}, 97 \%\right)$ were purchased from Strem and filtered through a $0.2 \mu \mathrm{m}$ PTFE syringe filter before use.

\section{CdSe Cores}

CdSe cores used for overcoating were synthesized according to a method used in the literature $^{1}$ but scaled up with twice the amount of chemicals. $120 \mathrm{mg} \mathrm{CdO}, 560 \mathrm{mg}$ octadecylphosphonic acid (ODPA) and 6 g trioctylphosphine oxide (TOPO) were added to a 100 mL flask. The mixture was heated to $150{ }^{\circ} \mathrm{C}$ and degassed under vacuum for 1 hour. Under nitrogen flow, the reaction mixture was heated to $330{ }^{\circ} \mathrm{C}$ to form a clear solution. After adding $3.0 \mathrm{~mL}$ trioctylphosphine (TOP) to the solution, the temperature was brought up to $380^{\circ} \mathrm{C}$, at which point Se/TOP (120 mg Se in $1.0 \mathrm{~mL}$ TOP) solution was swiftly injected into the flask. When the CdSe core nanocrystals reached the desired size, the reaction was terminated by removing the heat. $10 \mathrm{~mL}$ hexane was added to the resulting reaction solution to form a stock solution. In Figure S1, we plot the ensemble absorption spectra, measured with a conventional UV-Vis spectrophotometer, of the different synthetic batches of CdSe cores. These CdSe cores were used in Figures 2, 3, 4, 5, and 6 of the main text.

For Figure 2 of the main text, aliquots were drawn during a single growth. This was performed by withdrawing a few microliters of the growth solution from the flask after injection and quenching the drawn solution in $\sim 1 \mathrm{~mL}$ of hexanes. After cleaning in hexanes, the same syringe was used to withdraw the next aliquot. All aliquots were stored at $5{ }^{\circ} \mathrm{C}$ 
until needed. In Figure S2, we plot the ensemble photoluminescence spectra, measured with a conventional fluorometer, of the aliquots of CdSe cores drawn from a single synthetic batch. These CdSe cores were used in Figure 2 of the main text.

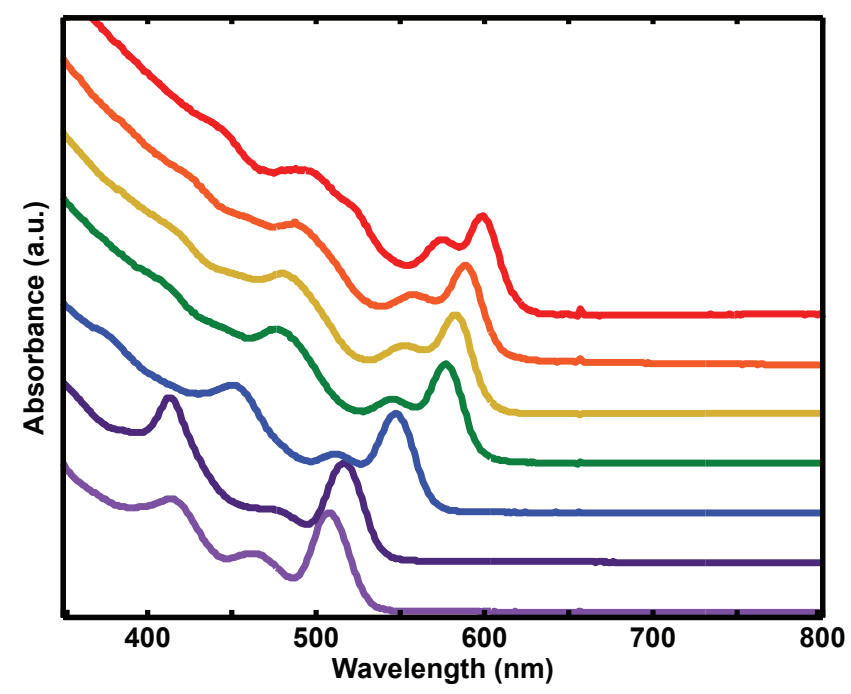

Figure S1: Ensemble absorption spectra of the different synthetic batches of CdSe cores. These CdSe cores were used in Figures 2, 3, 4, 5, and 6 of the main text.

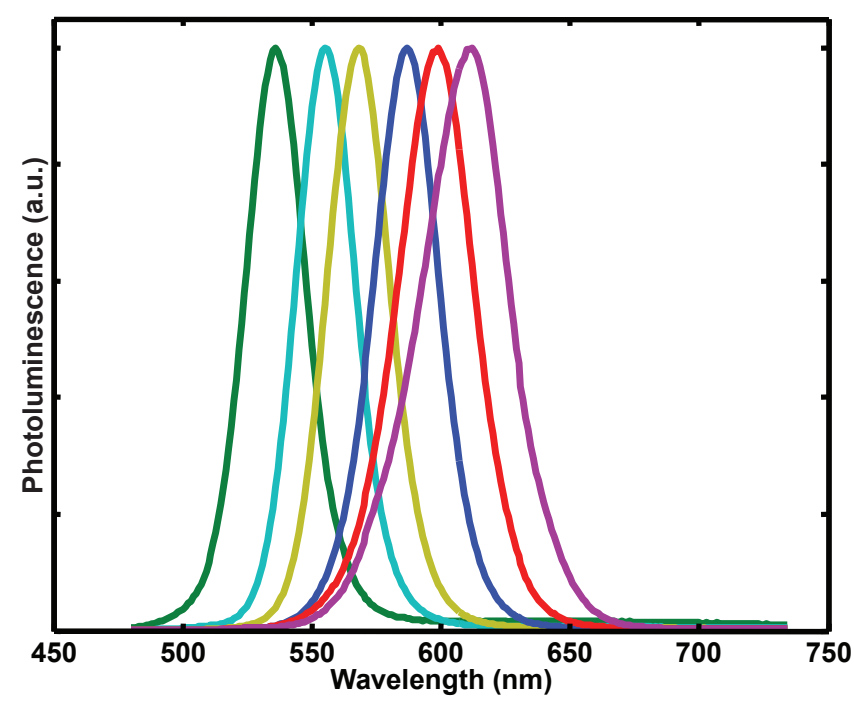

Figure S2: Ensemble photoluminescence spectra of CdSe cores drawn during a single synthetic batch. These CdSe cores were used in Figure 2 of the main text. 


\section{CdSe Overcoating}

CdSe core overcoating was performed using a modification of a procedure in the literature. ${ }^{2}$ CdSe cores that were synthesized as described above were purified through precipitation with acetone and methanol, centrifugation, removal of supernatant, and resuspension in hexanes. These cores were injected into $100 \mathrm{~mL}$ flask containing a solution of $5 \mathrm{~g}$ TOPO and 200 mg of n-hexylphosphonic acid that had been degassed under vacuum at $50{ }^{\circ} \mathrm{C}$. The solution containing the CdSe cores was then degassed under vacuum for 1 hour at $80{ }^{\circ} \mathrm{C}$. Under nitrogen, the temperature was increased to $140{ }^{\circ} \mathrm{C}$ and $0.5 \mathrm{~mL}$ of decylamine was added while stirring. $0.2 \mathrm{M}$ solutions of the shell precursors were prepared in an inert atmosphere by dissolving the appropriate amounts of each precursor into TOP. Two syringes were used for the injection: one for (TMS) $)_{2} \mathrm{~S}$ and the other for either $\mathrm{ZnEt}_{2}, \mathrm{CdMe}_{2}$, or both. The molar quantity of $\mathrm{ZnEt}_{2}$ or $\mathrm{CdMe}_{2}$ was calculated by considering the number of CdSe cores, the size of the cores, and the amount of material necessary for a monolayer of the shell. For $(\mathrm{TMS})_{2} \mathrm{~S}$, twice the molar amount was used. The precursor solutions were simultaneously injected into the flask at a rate such that $\sim 7$ monolayers were expected to be grown in 3 hours. Aliquots were drawn during shell growth as discussed above and stored similarly.

In Figure S3, we plot the ensemble photoluminescence spectra, measured using a conventional fluorometer, of the aliquots drawn during the shell overcoating. The results of S-PCFS performed on these aliquots are plotted in Figures 3, 4, and 6 of the main text. 

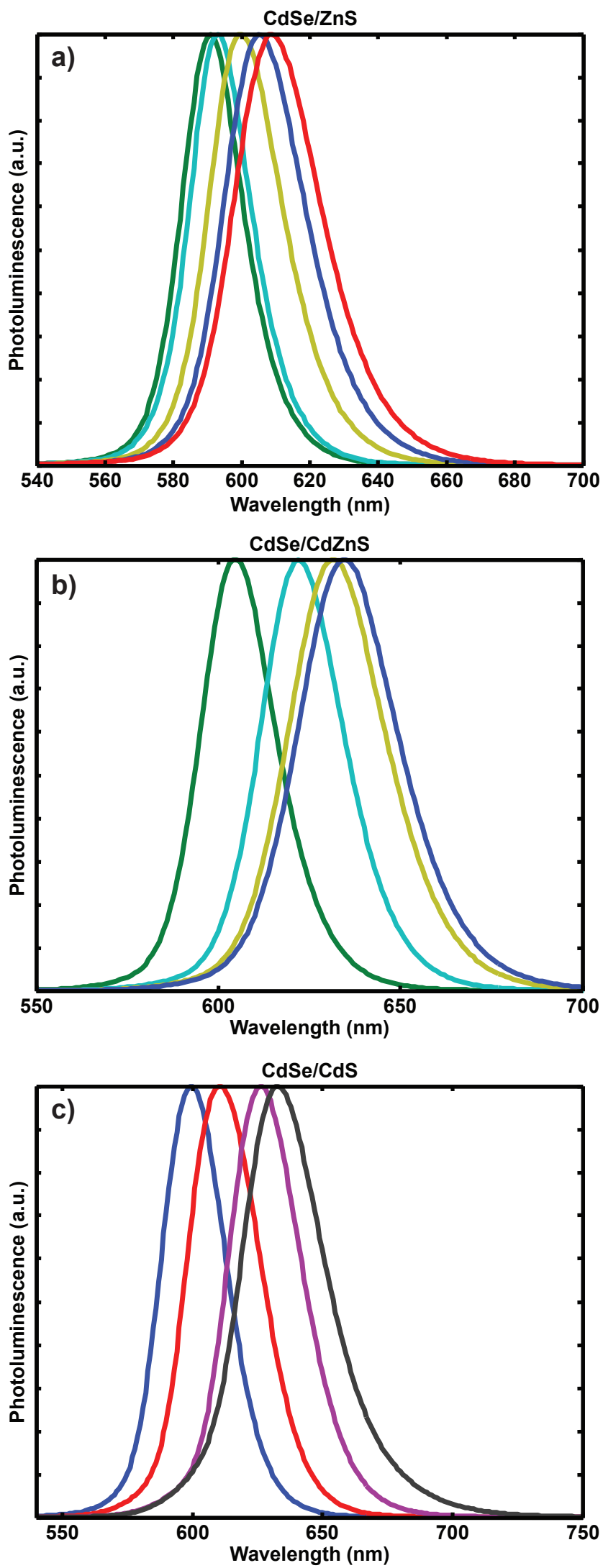

Figure S3: Ensemble photoluminescence spectra of a) CdSe/ZnS, b) CdSe/CdZnS, and c) $\mathrm{CdSe} / \mathrm{CdS}$ core/shell nanocrystals. The results of S-PCFS performed on these aliquots are plotted in Figures 3, 4, and 6 of the main text. 


\section{Sum of Two Boltzmann-Weighted Gaussians}

Here, we use a simple model to illustrate the effect that multiple emissive fine structure states may have on the linewidth of single-nanocrystal spectra. We first assume that there are two emissive fine structure states with the same spectra. For simplicity, we use two Gaussian functions, each with $50 \mathrm{meV}$ full-width at half-maximum (FWHM), and an energy separation dE. We can then calculate the spectrum as a sum of the two Gaussians while weighting the amplitude of the upper state using the Boltzmann factor $e^{\mathrm{dE} / \mathrm{k}_{\mathrm{B}} \mathrm{T}}$, where $\mathrm{k}_{\mathrm{B}} \mathrm{T}$ is the available thermal energy.
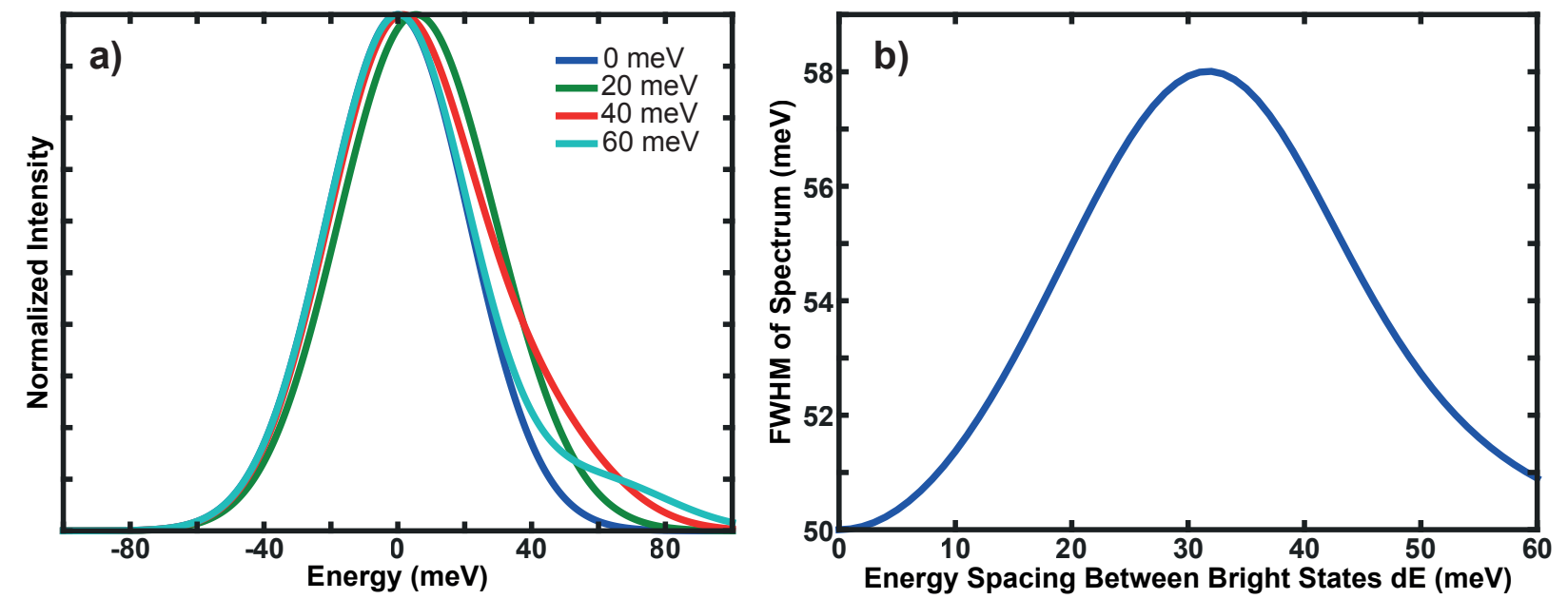

Figure S4: a) For different energy separations dE, we plot the sum of the two Boltzmann-weighted Gaussians. We see that the spectrum broadens initially with increasing dE, but at much larger splittings, the upper state contributes more as a shoulder. b) The FWHM of the sum of the two Gaussians initially increases with increasing $\mathrm{dE}$ but then decreases.

From Figure S4, we see that emission from fine-structure states can contribute a small, but non-negligible amount to the overall single-nanocrystal spectrum. In the case of Gaussian spectra with $50 \mathrm{meV}$ FWHM, we expect a contribution of no more than $\sim 8 \mathrm{meV}$. 


\section{Comparison of Wurtzite and Zincblende Particles}

Zincblende CdSe cores were synthesized using a literature method. ${ }^{3}$ Their first absorption feature was at $578 \mathrm{~nm}$. ZnS overcoating was performed using the same procedure as above. We compare these particles to the CdSe particles with first absorption feature at $577 \mathrm{~nm}$ shown in Figure 3 of the main text. We compare the core/shell particles with approximately the same thickness of $\mathrm{ZnS}$ shell.

In Figure S5, we show the single-nanocrystal spectral correlation function $p^{\text {single }}(\zeta)$ measured from S-PCFS for these particles. We can see that there is essentially no difference between the two spectral correlation functions. The effective single-NC spectral linewidths, obtained through fitting, ${ }^{4}$ are also nearly identical (52 meV vs. $\left.53 \mathrm{meV}\right)$.

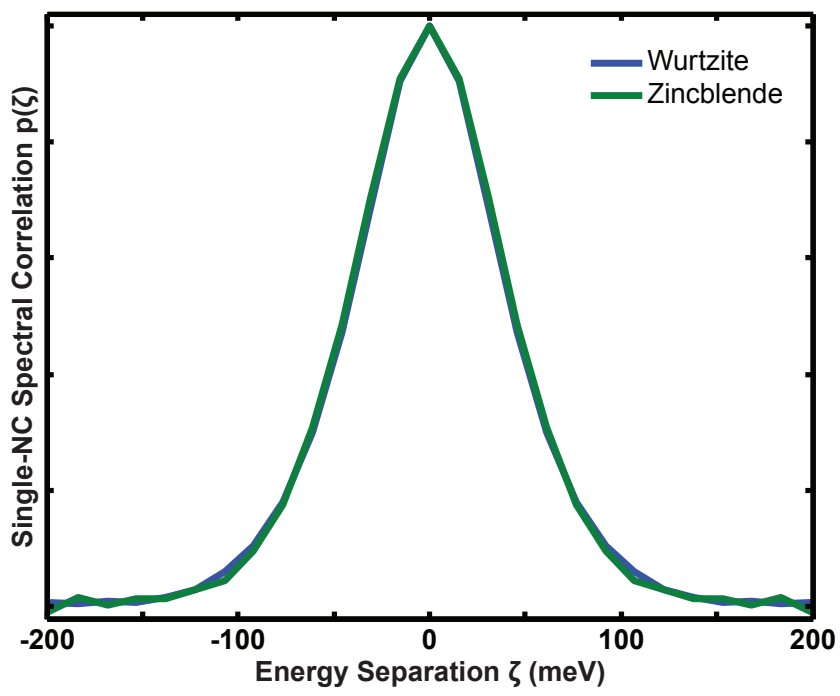

Figure S5: Wurtzite and zincblende CdSe cores were synthesized with nearly the same energy of the first absorption feature (577 $\mathrm{nm}$ and $578 \mathrm{~nm}$ respectively). The particles were then overcoated with $\mathrm{ZnS}$. The single-NC spectral correlation functions $p^{\text {single }}(\zeta)$ measured by S-PCFS are shown for the two particles with approximately 5 monolayers of $\mathrm{ZnS}$ shell. 


\section{References}

(1) Chen, O.; Zhao, J.; Chauhan, V. P.; Cui, J.; Wong, C.; Harris, D. K.; Wei, H.; Han, H.-S.;

Fukumura, D.; Jain, R. K.; Bawendi, M. G. Nat. Mater. 2013, 12, 445-451.

(2) Liu, W.; Howarth, M.; Greytak, A. B.; Zheng, Y.; Nocera, D. G.; Ting, A. Y.; Bawendi, M. G. J. Am. Chem. Soc. 2008, 130, 1274-1284.

(3) Chen, O.; Yang, Y.; Wang, T.; Wu, H.; Niu, C.; Yang, J.; Cao, Y. C. J. Am. Chem. Soc. 2011, 133, 17504-17512.

(4) Cui, J.; Beyler, A. P.; Marshall, L. F.; Chen, O.; Harris, D. K.; Wanger, D. D.; Brokmann, X.; Bawendi, M. G. Nat. Chem. 2013, 5, 602-606. 